\title{
Atomic correlations in itinerant ferromagnets: quasi-particle bands of nickel
}

\author{
J. Bünemann ${ }^{1}$, F. Gebhard ${ }^{2}$, T. Ohm ${ }^{3}$, R. Umstätter ${ }^{3}$, S. Weiser ${ }^{3}$, W. Weber ${ }^{3}$, \\ R. Claessen ${ }^{4}$, D. $\mathrm{Ehm}^{5}$, A. Harasawa ${ }^{6}$, A. Kakizaki ${ }^{6}$, A. Kimura ${ }^{7}$, G. Nicolay ${ }^{5}$, S. Shin ${ }^{6}$, and V.N. Strocov ${ }^{4}$ \\ ${ }^{1}$ Oxford University, Physical and Theoretical Chemistry Laboratory, Oxford OX1 3QZ, United Kingdom \\ ${ }^{2}$ Fachbereich Physik, Philipps-Universität Marburg, D-35032 Marburg, Germany \\ ${ }^{3}$ Institut für Physik, Universität Dortmund, D-44221 Dortmund, Germany \\ ${ }^{4}$ Experimentalphysik II, Universität Augsburg, D-86135 Augsburg, Germany \\ ${ }^{5}$ Fachrichtung Experimentalphysik, Universität des Saarlandes, D-66041 Saarbrücken, Germany \\ ${ }^{6}$ Institute for Solid-State Physics, The University of Tokyo, Kashiwa, Chiba 277-8581, Japan \\ 7 Dept. of Physical Sciences, Graduate School of Science, Hiroshima University, Higashi, Hiroshima 739-8526, Japan
}

\begin{abstract}
We measure the band structure of nickel along various high-symmetry lines of the bulk Brillouin zone with angle-resolved photoelectron spectroscopy. The Gutzwiller theory for a nine-band Hubbard model whose tight-binding parameters are obtained from non-magnetic density-functional theory resolves most of the long-standing discrepancies between experiment and theory on nickel. Thereby we support the view of itinerant ferromagnetism as induced by atomic correlations.
\end{abstract}

PACS numbers: 71.20.Be, 71.10.Fd, 71.10.Ay

Introduction. Studies of the electronic structure of ferromagnetic $\mathrm{Ni}$, in particular by angle-resolved photoelectron spectroscopy (ARPES), have since long revealed large discrepancies between experiments and results from spin-density-functional theory (SDFT) [1, 2, 3, 4, The width of the occupied part of the $3 d$ bands is approximately $W_{3 d}=3.3 \mathrm{eV}$, 5, [6], whereas all SDFT calculations yield values of $W_{\mathrm{SDFT}}=4.5 \mathrm{eV}$ [3]. In SDFT the exchange splitting of majority $(\uparrow)$ and minority $(\downarrow)$ spin bands is large and rather isotropic whereas the ARPES results report small and highly anisotropic splittings. Typical is the problem of the $X_{2 \downarrow}$ state which is positioned at $0.04 \mathrm{eV}$ below the Fermi energy $E_{\mathrm{F}}$ by the ARPES data. In contrast, all SDFT calculations predict the $X_{2 \downarrow}$ state to lie above $E_{\mathrm{F}}$; so does a study using the GW approximation [7]. As a consequence, a Fermi surface is predicted with two hole ellipsoids around the $X$ point whereas only one has been found in de Haas-van Alphen experiments [8], as later confirmed by ARPES [田, 司, 目].

Here, we present precise ARPES data on the electronic structure of $\mathrm{Ni}$, in which full control over the three-dimensional wave vector $\mathbf{k}$ was achieved using very low-energy electron diffraction (VLEED) and ARPES [9]. For comparison we use the quasi-particle bands from Gutzwiller theory [10, 11] which is based on a multi-band Hubbard model whose electron-transfer amplitudes are derived from non-magnetic DFT calculations. We obtain good agreement with our ARPES data whereby we resolve for the first time all of the qualitative discrepancies between experiment and SDFT for the band structure of $\mathrm{Ni}$. We argue that the DFT calculations underestimate the ratio between the partial charge densities in the $4 s p$ and the $3 d$ bands. This appears to be a general problem for DFT treatments of transition metals, see Ref. 12 .

Experiment. ARPES is a well-established tool for the measurement of quasi-particle dispersions $E(\mathbf{k})$ in crys- talline solids. However, a principal problem concerns the determination of the surface-perpendicular wave vector component $k_{\perp}$ of the photoelectron which is changed when the photoelectron exits into the vacuum. Full control of the three-dimensional wave vector $\mathbf{k}$ within the solid can be regained, if the dispersion of the photoelectron final states is known. The free-electron approximation which is usually employed for this purpose [5, 6, 13] frequently fails because the final-state bands are generally far more complicated [9].

A precise determination of both quasi-particle energies and wave vectors is essential for a detailed comparison between experiment and theory. To this end, we have controlled $\mathbf{k}$ using the following route. First, angledependent VLEED is applied to determine the unoccupied states above the vacuum level whose momentum $\mathbf{k}$ lies on surface-parallel high-symmetry lines of the Brillouin zone (BZ), relying on the fact that the photoelectron final states can be viewed as time-reversed LEED states. Photoemission via these states, employing the Constant-Final-State (CFS) mode, is then used to map out the dispersion of the occupied quasi-particle bands along these lines. The usefulness and accuracy of this method has previously been demonstrated for $\mathrm{Cu}$ [9].

In this work we report on measurements of the $\mathrm{Ni}(110)$ surface. The VLEED experiment was performed with a conventional LEED unit operated in the retarding field mode. ARPES in the CFS mode was carried out at beamline 18-A of the Photon Factory (Tsukuba, Japan); see Ref. 9 for further details. Both VLEED and ARPES measurements were performed along the $\overline{\Gamma X}, \overline{\Gamma Y}$, and $\overline{\Gamma S}$ azimuths of the (110) surface, giving access to the band dispersions along the $\Gamma K X, \Gamma X$, and $X L$ lines of the bulk Brillouin zone. The ARPES data on the quasiparticle dispersions are presented in Fig. 1 1 as a gray-scale map of the negative second derivative of the photocurrent [9]. Critical point energies are given in Table [. 


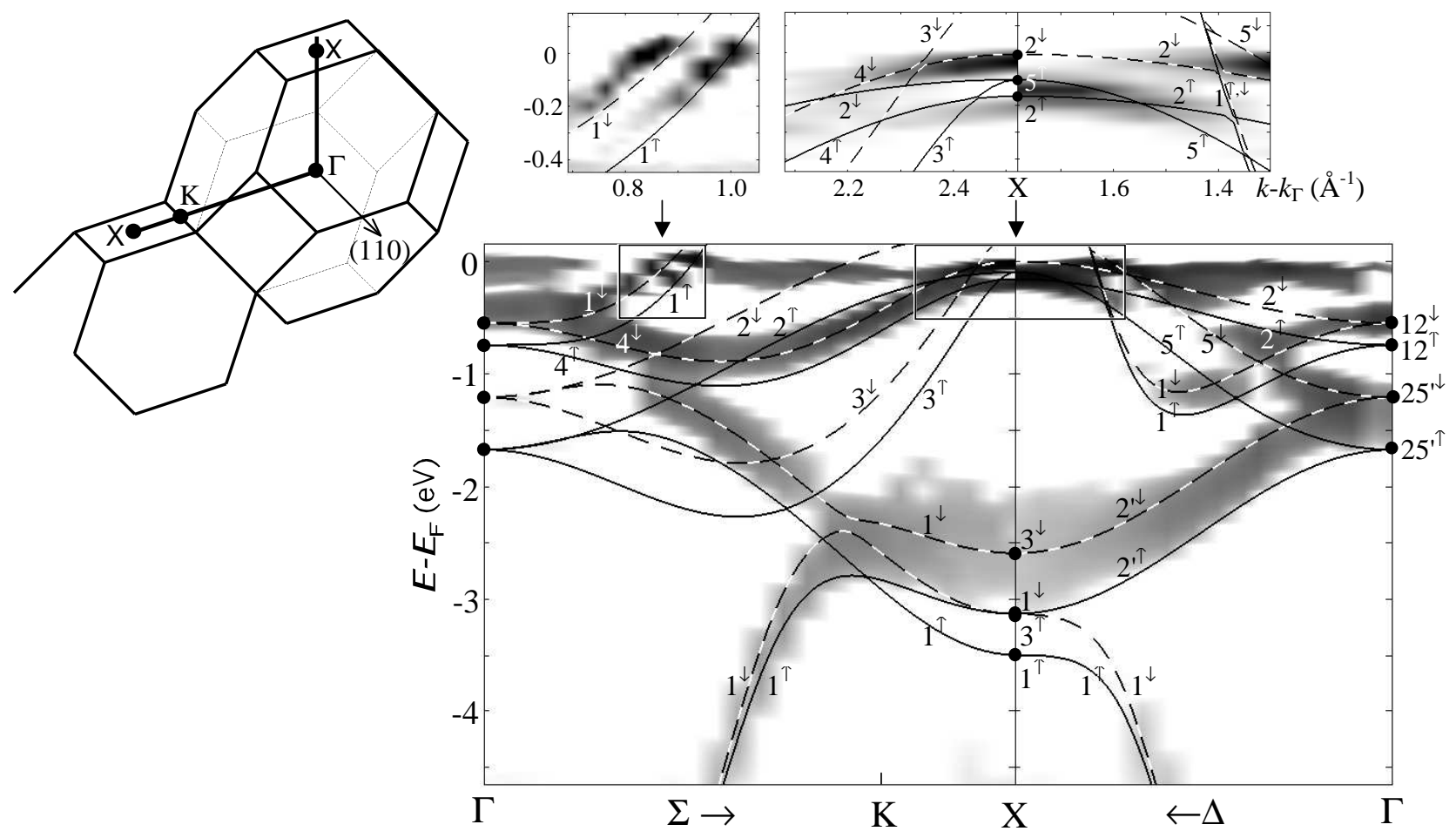

FIG. 1: Grey-scale plot of the negative second derivative of the ARPES intensity for nickel with respect to energy, $-d^{2} I / d E^{2}$, on a logarithmic scale (insets: linear scale) for the $\Gamma K X$ and $\Gamma X$ directions of the BZ. The dispersionless structure at $E_{F}$ is due to a residual Fermi edge resulting from indirect transitions. Some bands $\left(\Delta_{2}, X_{1}, \Sigma_{2}, \Sigma_{3}, \Sigma_{1}\right.$ towards $\left.\Gamma\right)$ are not seen due to unfavorable matrix elements, depending on geometry and chosen final state. Theoretical curves are G-DFT III, see table II.

TABLE I: Binding energies in $\mathrm{eV}$ with respect to the Fermi energy $E_{\mathrm{F}}(>0$ for occupied states). $\langle\ldots\rangle$ indicates the spin average, error bars in the experiments without spin resolution are given as \pm . Theoretical data show the spin average and the exchange splittings in square brackets. The second column denote the orbital character of the states, $t \equiv t_{2 g}, e \equiv e_{g}$, capital letters: dominant character. The spin-polarized data $\left\langle\Lambda_{3 ; f}\right\rangle$ were taken at fractions $f$ of the $\Gamma L$ distance, with the emphasis on the analysis of the exchange splittings. For all calculations, including the SDFT, a spin-only moment of $\mu=0.55 \mu_{\mathrm{B}}$ was used. G-DFT I: $A=12 \mathrm{eV}, n_{d}=8.8727$; G-DFT II: $A=9 \mathrm{eV}, n_{d}=8.8727$; G-DFT III: $A=9 \mathrm{eV}, n_{d}=8.7793$.

\begin{tabular}{|c|c|c|c|c|c|c|c|}
\hline Symmetry & Character & Experiment & Reference & G-DFT I & G-DFT II & G-DFT III & SDFT \\
\hline$\left\langle\Gamma_{1}\right\rangle$ & $\mathrm{S}$ & $8.90 \pm 0.30$ & & 8.87 & 8.89 & 8.86 & $8.96[-0.11]$ \\
\hline$\left\langle\Gamma_{25^{\prime}}\right\rangle$ & $\mathrm{T}$ & $1.30 \pm 0.06$ & & $1.34[0.62]$ & $1.52[0.62]$ & $1.44[0.46]$ & $1.99[0.43]$ \\
\hline$\left\langle\Gamma_{12}\right\rangle$ & $\mathrm{E}$ & $0.48 \pm 0.08$ & & $0.62[0.28]$ & $0.71[0.29]$ & $0.65[0.195]$ & $0.86[0.41]$ \\
\hline$\left\langle X_{1}\right\rangle$ & $\mathrm{sE}$ & $3.30 \pm 0.20$ & 6 & $2.98[0.57]$ & $3.39[0.50]$ & $3.31[0.36]$ & $4.37[0.20]$ \\
\hline$\left\langle X_{3}\right\rangle$ & $\mathrm{T}$ & $2.63 \pm 0.10$ & & $2.60[0.78]$ & $2.95[0.72]$ & $2.86[0.54]$ & $3.82[0.37]$ \\
\hline$X_{2 \uparrow}$ & $\mathrm{E}$ & $0.21 \pm 0.03$ & & 0.23 & 0.27 & 0.165 & 0.35 \\
\hline$X_{2 \downarrow}$ & $\mathrm{E}$ & $0.04 \pm 0.03$ & & 0.02 & 0.02 & 0.01 & -0.09 \\
\hline$X_{5 \uparrow}$ & $\mathrm{T}$ & $0.15 \pm 0.03$ & & 0.22 & 0.24 & 0.10 & 0.23 \\
\hline$\Delta_{e_{g}}\left(X_{2}\right)$ & $\mathrm{E}$ & $0.17 \pm 0.05$ & & 0.214 & 0.25 & 0.155 & 0.44 \\
\hline$\Delta_{t_{2 g}}\left(X_{5}\right)$ & $\mathrm{T}$ & $0.33 \pm 0.04$ & 5 & 0.45 & 0.49 & 0.38 & 0.56 \\
\hline$\left\langle K_{2}\right\rangle$ & spTe & $2.48 \pm 0.06$ & & $2.33[0.70]$ & $2.64[0.66]$ & $2.59[0.50]$ & $3.37[0.32]$ \\
\hline$\left\langle K_{4}\right\rangle$ & $\mathrm{pE}$ & $0.47 \pm 0.03$ & & $0.51[0.26]$ & $0.58[0.28]$ & $0.51[0.185]$ & $0.70[0.41]$ \\
\hline$\left\langle L_{1}\right\rangle$ & sT & $3.66 \pm 0.10$ & & $3.21[0.76]$ & $3.59[0.68]$ & $3.51[0.515]$ & $4.56[0.23]$ \\
\hline$\left\langle L_{3}\right\rangle$ & $\mathrm{tE}$ & $1.43 \pm 0.07$ & & $1.39[0.48]$ & $1.58[0.47]$ & $1.51[0.34]$ & $2.02[0.40]$ \\
\hline$L_{3 \uparrow}$ & $\mathrm{Te}$ & $0.18 \pm 0.03$ & & $0.30[0.37]$ & $0.34[0.41]$ & $0.215[0.30]$ & $0.38[0.50]$ \\
\hline$\left\langle L_{2^{\prime}}\right\rangle$ & $\mathrm{P}$ & $1.00 \pm 0.20$ & 6 & 0.32 & $0.29[0.0]$ & $0.97[0.0]$ & $0.24[-0.12]$ \\
\hline$\left\langle W_{1}\right\rangle$ & $\mathrm{sE}$ & $0.65 \pm 0.10$ & 6] & $0.66[0.28]$ & $0.76[0.30]$ & $0.69[0.20]$ & $0.94[0.39]$ \\
\hline$W_{1^{\prime} \uparrow}$ & $\mathrm{T}$ & $0.15 \pm 0.10$ & 6 & $0.23[0.46]$ & $0.25[0.52]$ & $0.11[0.38]$ & $0.23[0.56]$ \\
\hline$\left\langle\Lambda_{3 ; 1 / 3}\right\rangle$ & $\mathrm{ptE}$ & $0.57[0.16 \pm 0.02]$ & 14 & $0.64[0.30]$ & $0.73[0.32]$ & $0.67[0.22]$ & $0.90[0.42]$ \\
\hline$\left\langle\Lambda_{3 ; 1 / 2}\right\rangle$ & $\mathrm{ptE}$ & $0.50[0.21 \pm 0.02]$ & 14 & $0.54[0.34]$ & $0.61[0.37]$ & $0.55[0.26]$ & $0.76[0.44]$ \\
\hline$\left\langle\Lambda_{3 ; 2 / 3}\right\rangle$ & pTE & $0.35[0.25 \pm 0.02]$ & 14 & $0.35[0.37]$ & $0.39[0.41]$ & $0.33[0.29]$ & $0.49[0.48]$ \\
\hline
\end{tabular}


Gutzwiller theory. The Gutzwiller theory employs a tight-binding Hamiltonian for a basis of $4 s, 4 p$ and $3 d$ orbitals

$$
\widehat{H}_{1}=\sum_{i \neq j=1 ; \sigma, \sigma^{\prime}}^{L} t_{i, \sigma ; j, \sigma^{\prime}} \widehat{c}_{i ; \sigma}^{+} \widehat{c}_{j ; \sigma^{\prime}}+\sum_{i ; \sigma} \widetilde{\epsilon}_{\sigma} \widehat{c}_{i ; \sigma}^{+} \widehat{c}_{i ; \sigma} .
$$

The energy-transfer integrals $t_{i, \sigma ; j, \sigma^{\prime}}$ between the spinorbitals $\sigma$ at site $i$ and $\sigma^{\prime}$ at site $j$ range up to third nearest neighbors. They result from a fit to DFT energy bands of non-magnetic $\mathrm{Ni}$, calculated with the LAPWWIEN code [15] and using the local-density approximation; for details of the fit procedure, see Ref. 16. A value of $15 \mathrm{meV}$ for the root-mean-square deviation was obtained for all bands up to $2 \mathrm{eV}$ above the Fermi energy. We note that the DFT energy of the $4 p$-type $L_{2^{\prime}}$ state is $0.120 \mathrm{eV}$ below $E_{\mathrm{F}}$, and the bare $3 d$ band width in our tight-binding model is $W_{\text {bare }} \approx W_{\mathrm{DFT}}=4.5 \mathrm{eV}$.

The single-particle term is supplemented by the atomic Coulomb interactions among the $3 d$ electrons,

$$
\begin{aligned}
\widehat{H} & =\widehat{H}_{1}+\sum_{i} \widehat{H}_{i ; \text { at }}, \\
\widehat{H}_{i ; \text { at }} & =\sum_{\sigma_{1}, \sigma_{2}, \sigma_{3}, \sigma_{4}} \mathcal{U}^{\sigma_{1}, \sigma_{2} ; \sigma_{3}, \sigma_{4}} \widehat{c}_{i ; \sigma_{1}}^{+} \widehat{c}_{i ; \sigma_{2}}^{+} \widehat{c}_{i ; \sigma_{3}} \widehat{c}_{i ; \sigma_{4}} \\
& =\sum_{\Gamma} E_{\Gamma} \widehat{m}_{i ; \Gamma} .
\end{aligned}
$$

Here, $\widehat{m}_{i ; \Gamma}$ is the projector onto the atomic eigenstate $|\Gamma\rangle_{i}$ with energy $E_{\Gamma}$. In the spherical approximation, the Coulomb parameters $\mathcal{U}^{\sigma_{1}, \sigma_{2} ; \sigma_{3}, \sigma_{4}}$ can be obtained from three Racah parameters. In this work we choose $B=$ $0.09 \mathrm{eV}$ and $C=0.40 \mathrm{eV}$, guided by spectroscopic data for isolated $\mathrm{Ni}^{2+}$ and $\mathrm{Ni}^{3+}$ ions; see Table 5.1 of Sugano et al. [17]. This choice fulfills the 'canonical' ratio $C / B \approx$ 4.5 , found for transition metal ions both in atomic and impurity spectroscopy [17]. The Racah parameter $A=$ $\mathcal{O}(10 \mathrm{eV})$ is adjusted; see below.

The sizable atomic Coulomb interactions suppress local charge fluctuations [18] which occur in the limit of independent particles. Therefore, Gutzwiller [19] proposed to address the states

$$
\left|\Psi_{\mathrm{G}}\right\rangle=\widehat{P}_{\mathrm{G}}|\Phi\rangle \quad, \quad \widehat{P}_{\mathrm{G}}=\prod_{i ; \Gamma} \eta_{\Gamma}^{\widehat{m}_{i ; \Gamma}}
$$

as a variational approximation for the true ground state of $\widehat{H}$. The real numbers $\eta_{\Gamma}$ parameterize the Gutzwiller correlator $\widehat{P}_{\mathrm{G}}$ which reduces energetically unfavorable atomic configurations in the normalized one-particle product state $|\Phi\rangle$. For an open $d$-shell system, there are $2^{10}$, i.e., of the order of $10^{3}$ variational parameters.

The variational ground state is determined by a minimization of the expectation value $\langle\widehat{H}\rangle_{\Psi_{\mathrm{G}}}$ with respect to the 'internal' parameters $\eta_{\Gamma}$ and the wave function $|\Phi\rangle$. In infinite dimensions [10, 11] this leads to the condition that $|\Phi\rangle$ is the ground state of the effective Hamiltonian

$$
\begin{aligned}
\widehat{H}^{\mathrm{eff}} & =\sum_{\mathbf{k}, \sigma, \sigma^{\prime}} \widetilde{S}_{\sigma, \sigma^{\prime}}(\mathbf{k}) \widehat{c}_{\mathbf{k}, \sigma}^{+} \widehat{c}_{\mathbf{k}, \sigma^{\prime}}, \\
\widetilde{S}_{\sigma, \sigma^{\prime}}(\mathbf{k}) & =\sqrt{q_{\sigma}} \sqrt{q_{\sigma^{\prime}}} \epsilon_{\sigma, \sigma^{\prime}}(\mathbf{k})+\delta_{\sigma, \sigma^{\prime}}\left(\widetilde{\epsilon}_{\sigma}+\lambda_{\sigma}\right),
\end{aligned}
$$

where $\epsilon_{\sigma, \sigma^{\prime}}(\mathbf{k})$ is the Fourier transform of the bare electron-transfer integrals $t_{i, \sigma ; j, \sigma^{\prime}}$. The spin-orbital energy shifts $\lambda_{\sigma}$ act as 'external' variational parameters since they determine $|\Phi\rangle$ via the eigenvalue equation $\left(\widehat{H}^{\text {eff }}-E\right)|\Phi\rangle=0$.

The quasi-particle excitations from energy bands given by the eigenvalues $E(\mathbf{k}, \gamma)$ of $\widehat{H}^{\text {eff }} 11$. Note, that the effective single-particle Hamiltonian differs from $\widehat{H}_{1}$ by the renormalization factors $0 \leq q_{\sigma} \leq 1$ and the energy shifts $\lambda_{\sigma}$. Therefore, the bare bands of $\widehat{H}_{1}$ become narrowed and mixed in the quasi-particle excitations of the correlated system. The above results are strictly valid only for $D=\infty$. However, $1 / D$ corrections were found to be small for $D=3$ single-band Fermi-liquid systems [11].

The Gutzwiller method overestimates the optimum magnetic moment by about $10 \%$ to $15 \%$; for a remedy of this problem, see Ref. 20. Therefore, we work with a fixed magnetic moment $\mu=0.55 \mu_{\mathrm{B}}$, the experimental spinonly moment. Spin-orbit coupling has not yet been incorporated into our Gutzwiller codes as this requires a major reprogramming effort. We keep the partial densities $n_{s}$, $n_{p}$, and $n_{d}$ fixed to the values obtained from the DFT one-particle Hamiltonian. We have studied other variants of applying the Gutzwiller-DFT which allow charge flow between $4 s p$ and $3 d$ channels, yet the effects on the quasi-particle bands are found to be small [20].

Results for nickel. In cubic iron-group metals and under the limitations of keeping $n_{d}, n_{s}$, and $n_{p}$ fixed, symmetry allows for three independent energy shifts $\lambda_{\sigma}$ for the $3 d$ electrons. They govern the exchange splittings $\Delta\left(t_{2 g}\right)$ and $\Delta\left(e_{g}\right)$, and the crystal-field splitting $\Delta\left(e_{g} / t_{2 g}\right)$. For $\mathrm{Ni}$, the exchange splittings of the $4 s$ and $4 p$ orbitals turn out to be of very minor importance. Therefore, we minimize the variational ground-state energy for a fixed magnetic moment $\mu$ with respect to three external and about 400 internal variational parameters.

We find the best agreement with the occupied $3 d$ band width $W_{3 d}=3.3 \mathrm{eV}$ at $X_{1}$ for a value $A=9 \mathrm{eV}$, see table If, column G-DFT II. Then, the condensation energy, i.e., the energy gain for the ferromagnetic phase as compared to the paramagnetic one, is $E_{\text {cond }} \approx$ $40 \mathrm{meV}=0.8 k_{\mathrm{B}} T_{\mathrm{C}}\left(T_{\mathrm{C}}=630 \mathrm{~K}\right.$ is the Curie temperature). This value of $A$ is much larger than in other multi-band studies [21, 22. In [21], only $3 d$ bands were incorporated. If we eliminate in our calculations the rather large $4 s p$ - $3 d$ hybridizations we also find that values of $A=2 \mathrm{eV} \ldots 3 \mathrm{eV}$ give ferromagnetic solutions with $E_{\text {cond }}=40 \mathrm{meV}$ and $W_{3 d} \approx 3 \mathrm{eV}$. The results of [22, where the $4 s p$ bands have also been included, are at variance with our findings. 
For all three G-DFT calculations of table $\mathbb{\text { If }}$ the energy of the state $X_{2 \downarrow}$ is pinned slightly below the Fermi energy, in agreement with experiment. This is an essential improvement over SDFT and even SDFT-GW calculations [7] which predict a second hole pocket at the $X$ point. Even a drastic change in the Racah parameter $A$ from $A_{\text {II }}=A_{\text {III }}=9 \mathrm{eV}$ to $A_{\mathrm{I}}=12 \mathrm{eV}$ does not change much the basic features of the Gutzwiller quasiparticle band structure. The pinning of $X_{2 \downarrow}$ is caused by the small variational parameter $\Delta\left(e_{g}\right)$, which in turn leads to a large fraction of $t_{2 g}$ holes in the minority spin bands, produced by a relatively large $\Delta\left(t_{2 g}\right)$. Because of the large nearest-neighbor hopping between $t_{2 g}$ orbitals, it is energetically favorable to generate as many $t_{2 g}$ holes in the minority spin bands as possible.

A more detailed inspection of the table indicates several significant discrepancies between experiment and the results from G-DFT II. Most notably, the energy of the state $L_{2^{\prime}}, E_{\mathrm{GII}}\left(L_{2^{\prime}}\right)=-0.3 \mathrm{eV}$, is much higher than in experiment, $E\left(L_{2^{\prime}}\right)=-1.0 \mathrm{eV}$. This discrepancy is caused by the underlying DFT calculation (see also table [1, column SDFT), as the $4 p$-type $L_{2^{\prime}}$ state is basically not affected by correlations in the $3 d$ shell. When we lower the bare $4 p$ level $\epsilon_{p}$ in (11) by $0.75 \mathrm{eV}$, we considerably improve the agreement with experiment especially for all states near the Fermi energy and thus also for the values of the exchange splittings. The photoelectron data reveal $\Delta_{e_{g}}\left(X_{2}\right)=170 \pm 50 \mathrm{meV}$ for the pure $d\left(e_{g}\right)$ state $X_{2}$, and $\Delta_{e_{g}}\left(X_{5}\right)=330 \pm 40 \mathrm{meV}$ [5] for the pure $d\left(t_{2 g}\right)$ state $X_{5}$, which agrees well with the data from the GDFT III. Lowering the $4 p$ level leads to a charge flow of about 0.1 electrons from $3 d$ to $4 p$ whereby the number of holes in the $3 d$ bands is significantly enhanced. Thus, there are more $3 d$ holes available to generate the magnetic moment, and the exchange splittings are reduced accordingly. Note that the G-DFT exchange splittings incr pase as a function of the binting energy by about $50 \%$, as ean be seen, e.g., from the values of the pure $t_{2 g}$ states $X_{5}, \Gamma_{25^{\prime}}$, and $X_{3}$. In contrast, they decrease in the SDFT because of the bigger orbital basis used there.

Conclusions. Hartree-Fock theory was the starting point for the Stoner-Slater band theories of itinerant ferromagnetism [23, 24]. For decades these theories have been the only ones to provide detailed comparison with experiments. In this paper we have shown for the prototypical ferromagnet $\mathrm{Ni}$ that, starting from a DFT-based one-particle Hamiltonian, the Gutzwiller theory can resolve the main discrepancies for Ni. We thereby confirm the qualitative findings of previous model studies [10, 21] on the basic mechanism of itinerant ferromagnetism. In $\mathrm{Ni}$ the $3 d^{8}, 3 d^{9}$, and $3 d^{10}$ multiplets are predominantly occupied to accommodate the $3 d$ electrons' itinerancy. However, the occupation of the low-lying $3 d^{8}$ spin triplets is significantly enhanced over the occupation of the $3 d^{8}$ singlets. As in magnetic insulators, Hund's first rule is found to be an important agent in itinerant ferromagnets.
Therefore, we corroborate early ideas by van Vleck [18, Gutzwiller [19] and others on the origin of itinerant ferromagnetism. In contrast to band aspects, they emphasized the importance of local moments in narrow-band metals as a consequence of strong atomic correlations.

Further predictions of our theory, e.g., the increase of the exchange splittings as a function of energy, should be tested by more detailed spin-resolved experiments.

W.W. thanks S.G. Louie and M.L. Cohen for their hospitality during his sabbatical stay. We thank H. Starnberg and P. Blaha for their help with the VLEED data acquisition and analysis. This work was supported by the Deutsche Forschungsgemeinschaft (446JAP/113/104/0, BU1309/2-1, CL124/5-1, WE1412/8-1).

[1] A. Goldmann, W. Gudat, and O. Rader in A. Goldmann (Ed), Landolt-Boernstein New Series Group III, Vol. 23C2 (Springer, Berlin, 1994).

[2] J. Callaway in P. Rhodes (Ed), Physics of Transition Metals 1980 (Conf. Ser. Notes 55, Inst. of Physics, Bristol, 1981), p. 1.

[3] V.L. Moruzzi, J.F. Janak, and A.R. Williams, Calculated Electronic Properties of Metals (Pergamon Press, New York, 1978).

[4] M. Donath, Surf. Sci. Rep. 20, 251 (1994).

[5] D.E. Eastman, F.J. Himpsel, and J.A. Knapp, Phys. Rev. Lett. 40, 1514 (1978).

[6] W. Eberhardt and E.W. Plummer, Phys. Rev. B 21, 3245 (1980).

[7] F. Aryasetiawan, Phys. Rev. B 46, 13051 (1992).

[8] D.C. Tsui, Phys. Rev. 164, 669 (1967).

[9] V.N. Strocov et al., Phys. Rev. Lett. 81, 4943 (1998); Phys. Rev. B 63, 205108 (2001).

[10] J. Bünemann, W. Weber, and F. Gebhard, Phys. Rev. B 57, 6896 (1998); Found. of Physics 30, 2011 (2000).

[11] J. Bünemann, F. Gebhard, and R. Thul, preprint condmat/0202434 (2002).

[12] The $p$-level energy in bcc Niobium had to be lowered by $0.65 \mathrm{eV}$ to make DFT Fermi surfaces agree best with experiment; see G.W. Crabtree et al., Phys. Rev. Lett. 42, 390 (1977).

[13] K. Ono et al., Solid State Comm. 107, 153 (1998).

[14] K.-P. Kämper, W. Schmitt, and G. Güntherodt, Phys. Rev. B 42, 10696 (1990).

[15] P. Blaha et al., Comp. Phys. Commun. 59, 399 (1990).

[16] W. Weber and L.F. Mattheiss, Phys. Rev. B 25, 2270 (1982).

[17] S. Sugano, Y. Tanabe, and H. Kamimura, Multiplets of Transition-Metal Ions in Crystals, Pure and Applied Physics 33 (Academic Press, New York, 1970).

[18] J.H. van Vleck, Rev. Mod. Phys. 25, 220 (1953).

[19] M.C. Gutzwiller, Phys. Rev. Lett. 10, 159 (1963).

[20] T. Ohm et al., J. Low Temp. Phys. 126, 1081 (2002).

[21] M.B. Zölfl et al., Phys. Rev. B 61, 12810 (2000).

[22] A.I. Lichtenstein, M.I. Katsnelson, and G. Kotliar, Phys. Rev. Lett. 87, 067205 (2001).

[23] J.C. Slater, Phys. Rev. 49, 537 (1936).

[24] E.C. Stoner, Proc. Roy. Soc. A 165, 372 (1938). 